\title{
Aplicativo com Realidade Aumentada para auxiliar no processo de ensino- aprendizagem de fundamentos de Mecânica Clássica
}

\author{
Guilherme B. Guimarães ${ }^{2}$, Adilmar C. Dantas ${ }^{1}$, Michel S. Xavier ${ }^{2}$, \\ Heidie S. Torres ${ }^{2}$, Kayro R. M. de Lima ${ }^{2}$, Sara L. Melo ${ }^{1}$, \\ Marcelo Z. do Nascimento ${ }^{1}$, Ricardo Kagimura ${ }^{2}$ \\ ${ }^{1}$ FACOM - Faculdade de Ciência da Computação \\ Universidade Federal de Uberlândia \\ Av. João Naves de Ávila, 2121-38408-100 Uberlândia, MG, Brasil \\ ${ }^{2}$ INFIS - Instituto de Física \\ Universidade Federal de Uberlândia \\ Av. João Naves de Ávila, 2121-38408-100 Uberlândia, MG, Brasil \\ email\{gui.brilhante, akanehar, miichelsx, heidietorres, kayrorogger, saraluziamelo, \\ marcelo.zanchetta, kagimura\}@gmail.com
}

\begin{abstract}
This paper presents the prototype of an application based on Augmented Reality (AR) to assist in the process of teaching and learning the fundamentals of Classical Mechanics, through theoretical and practical foundations made available digitally. The application is being developed based on observations made with students and teachers of the proposed discipline. The main objective of the prototype is that it functions as a study tool for students of the discipline and consequently that it contributes to improvements in the process of teaching and learning mediated by the use of technologies increasingly present in our daily lives.
\end{abstract}

Resumo. Este trabalho apresenta o protótipo de um aplicativo com base em Realidade Aumentada (RA) para auxiliar no processo de ensino-aprendizagem de fundamentos de Mecânica Clássica, por meio de fundamentos teóricos e práticos disponibilizados digitalmente. A aplicação está sendo desenvolvida com base em observações realizadas juntamente com alunos e professores da disciplina proposta. O principal objetivo do protótipo é que ele funcione como ferramenta de estudo para os alunos da disciplina e que consequentemente ele contribua com melhorias no processo de ensino e de aprendizagem intermediados pela utilização de tecnologias cada vez mais presentes em nosso cotidiano.

\section{Introdução}

A Física é uma disciplina geralmente considerada pelo alunos como abstrata e difícil de ser compreendida. De acordo com [McDermott 2001] isso ocorre devido às metas e realizações dos alunos, refletindo assim em uma lacuna entre eles e os professores. Para alguns alunos a principal dificuldade está no tradicionalismo metodológico, caracterizado pela abstração e ocasionando assim uma dificuldade para a compreensão correta dos fenômenos físicos [Kaufmann and Meyer 2008]. 
VIII Congresso Brasileiro de Informática na Educação (CBIE 2019)

Anais dos Workshops do VIII Congresso Brasileiro de Informática na Educação (WCBIE 2019)

De acordo com [Wieman and Perkins 2005] em suas pesquisas, ele demonstra a ineficácia da abordagem tradicional no ensino de Física, mostrando que o ensino tradicional está prejudicando a compreensão da disciplina pelos estudantes, pois os alunos estão memorizando os conceitos, fórmulas e receitas para a solução de problemas. Apesar dos esforços realizados pelos professores para alterar as metodologias e processos de ensino-aprendizagem, os meios encontrados pelos alunos para o estudo de Física acaba por fazê-la se tornar chata, enjoativa e irrelevante.

Um problema atualmente enfrentado nos cursos superiores de Física reflete no índice de evasão nas universidades públicas brasileiras. De acordo com [Barroso and Falcão 2004] isso ocorre devido a problemas acadêmicos expressados através de reprovações em disciplinas iniciais como Física 1 e Cálculo 1. Tendo em vista que, além dos reflexos desses problemas no Ensino Médio, levando os estudantes a chegarem no Ensino Superior com conhecimento insuficiente, há o fator desinteresse dos estudantes por cursos de baixa demanda.

Buscando reverter essas situações, alguns pesquisadores e educadores vêm procurando desenvolver soluções com o objetivo de tornar o processo de ensino e aprendizado de Física mais atrativo e proveitoso. Como exemplo, temos o trabalho [Dantas et al. 2017], em que autores desenvolveram e aplicaram um sistema de perguntas e respostas dinâmico, como o objetivo de tornar as aulas de Física mais descontraídas, permitindo assim uma melhor absorção do conteúdo didático por meio de jogo.

Os atuais avanços tecnológicos têm proporcionado uma grande expansão ao acesso de informações sobre os mais diversos assuntos. Dessa forma, integrar conteúdos de Física a essas novas ferramentas como, por exemplo, a realidade aumentada (RA) pode ser uma boa estratégia para buscar motivar estudantes no estudo dos conteúdos da disciplina. A grande maioria dos alunos possuem muito apreço por tecnologias como: jogos, celulares e computadores. Sendo assim, o uso de RA se apresenta como uma boa estratégia para auxiliar os alunos e professores no processo de ensino-aprendizagem de Física.

\section{Proposta do Aplicativo}

A RA permite criar percepções de inserção de objetos 3D no mundo real e a inclusão de sobreposições gráficas elaboradas pelo computador no mundo real [Azuma 1997]. De acordo com [Milgram and Kishino 1994], a realidade pode ser colocada em uma linha contínua que cresce conforme a quantidade de "mundo". Essa realidade é disponibilizada ao usuário, como exemplificado na Figura 1 .

Movendo-se da direita para a esquerda percebe-se o enfraquecimento virtual das realidades, sendo o extremo direito um ambiente totalmente virtual, e o extremo esquerdo o não uso dessa "virtualidade". Por isso afirma-se que a RA pode ser considerada uma boa ferramenta para realizar a transição de ambientes reais para os virtuais [Milgram and Kishino 1994].

Esse trabalho tem como objetivo o desenvolvimento de um aplicativo móvel usando a tecnologia de RA para auxiliar no processo de ensino-aprendizagem de conteúdos de Mecânica e de Matemática. Além disso, a aplicação pode auxiliar os professores a identificar em quais ementas disciplinares os alunos apresentaram mais dificuldades e as- 
VIII Congresso Brasileiro de Informática na Educação (CBIE 2019)

Anais dos Workshops do VIII Congresso Brasileiro de Informática na Educação (WCBIE 2019)

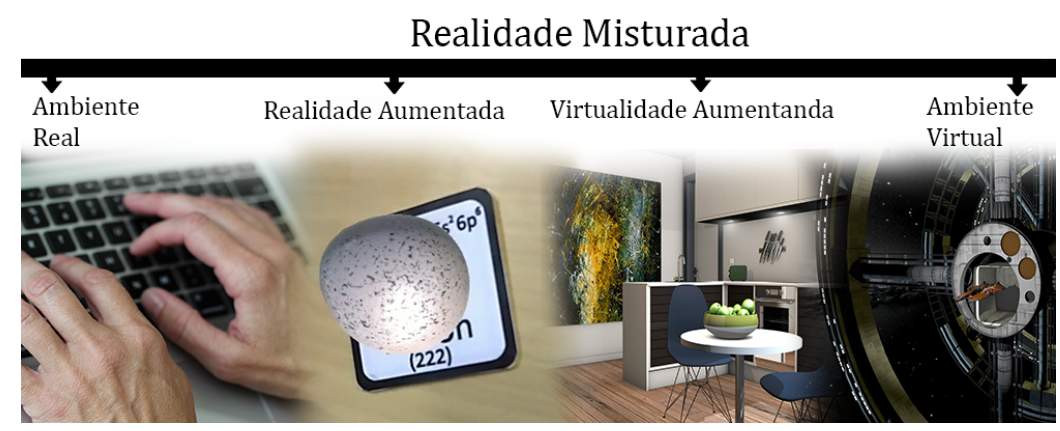

Figure 1. Exemplo do processo de transição do ambiente real para o virtual.

sim propor soluções para contornar a situação e consequentemente auxiliar na construção de uma melhor aprendizagem por parte dos alunos.

\subsection{Desenvolvimento do Aplicativo}

Para o desenvolvimento do aplicativo móvel foi utilizado softwares como o Vuforia e o Unity, uma plataforma que permite a criação de jogos e aplicativos em 2D e 3D, Realidade Virtual (RV) e RA, além de permitir o desenvolvimento para diversas plataformas eletrônicas como web, dispositivos móveis, consoles entre outros [Unity 2018]. O Vuforia é um Kit de Desenvolvimento de Software (SDK) que permite desenvolver aplicações em RV e RA para diferentes plataforma através do Unity [Vuforia 2018].

Para o desenvolvimento dos modelos tridimensionais (3D), foi necessária a utilização de dois softwares específicos: o Blender, um software de modelagem, animações, renderização e simulação que permite elaborar modelos com foco em desempenho de renderização e alta qualidade gráfica [Blender 2018]; e também o Google SketchUp, utilizado por muitos designers, arquitetos e engenheiros para a construção de maquetes e plantas baixas, sendo esse utilizado para a construção de modelos mais simples com desempenho de renderização [SketchUp 2018].

\section{Apresentação do Software}

O “Mecânica Clássica - Realidade Aumentada" é um aplicativo desenvolvido para dispositivos móveis com sistema operacional Android que, por meio da RA, permite aos alunos aprenderem e estudarem conceitos de Física 1 através de teorias e experimentos presentes na aplicação. $\mathrm{O}$ aplicativo está disponível através da Play Store por meio do seguinte endereço eletrônico https://play.google.com/store/apps/details?id=com.ufu.mecanica.

A interface desenvolvida para o aplicativo foi projetada para ser simples e altamente intuitiva, como na Figura 2 é apresentado as principais interfaces da aplicação. $\mathrm{Na}$ interface inicial são disponibilizadas as informações e curiosidades sobre a Mecânica Clássica, uma seção explicando sobre o funcionamento da RA, os marcadores para o usuário fazer download e por fim uma seção denominada Teoria, onde se encontram as seções com os tópicos de Física para serem estudadas através da RA.

Na Figura 3 temos um exemplo prático da utilização do aplicativo através da RA para abordar alguns tópicos de íFsica. Neste caso, está sendo abordado conceitos como decomposição de vetores e o uso da simetria do objeto para simplificar resoluções de 
VIII Congresso Brasileiro de Informática na Educação (CBIE 2019)

Anais dos Workshops do VIII Congresso Brasileiro de Informática na Educação (WCBIE 2019)
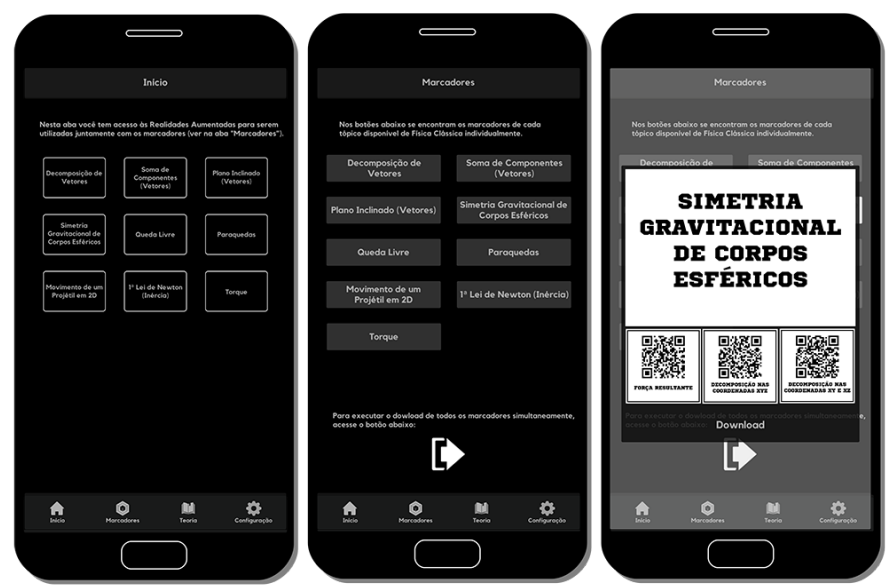

Figure 2. Interfaces do aplicativo: tela inicial, funcionalidades do RA e exemplos em RA.

problemas físicos, como no caso da força gravitacional devido a um objeto esférico. Para o correto funcionamento do sistema é necessário que a câmera do aparelho identifique os marcadores ou cartões que possibilitam habilitar a funcionalidade da realidade aumentada no dispositivo móvel.

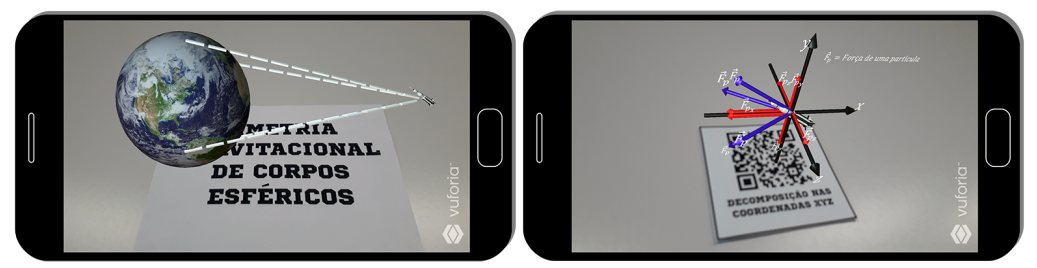

Figure 3. Exemplo da aplicação executando modelos conceituais de Física em RA.

\section{Resultados}

O aplicativo foi desenvolvido e submetido ao Comitê de Ética em Pesquisa de acordo com as conformidades da Universidade Federal de Uberlândia.Ele foi aplicado em duas turmas do primeiro ano do Ensino Médio, com um total de 51 alunos. A aplicação aconteceu juntamente com uma atividade denominada "Gincana de Vetores". Essa atividade teve como intuito estimular e avaliar os alunos quanto às operações vetoriais desenvolvidas em sala de aula com o auxilio do aplicativo. No fim da atividade os alunos foram submetidos a responder um questiona com as seguintes questões: você utilizou o aplicativo?, a aplicação te ajudou na aprendizagem dos conceitos envolvidos na atividade? Os resultados obtidos na aplicação dos questionários são apresentados no gráfico da Figura 4.

Analisando os gráficos da Figura 4 é possível concluir que $74,50 \%$ dos alunos utilizaram a aplicação para auxiliar na conclusão das atividades propostas. Além disso, foi possível observar que $84,31 \%$ dos alunos disseram que a aplicação realmente os auxiliou na aprendizagem dos conceitos envolvidos. 
VIII Congresso Brasileiro de Informática na Educação (CBIE 2019)

Anais dos Workshops do VIII Congresso Brasileiro de Informática na Educação (WCBIE 2019)

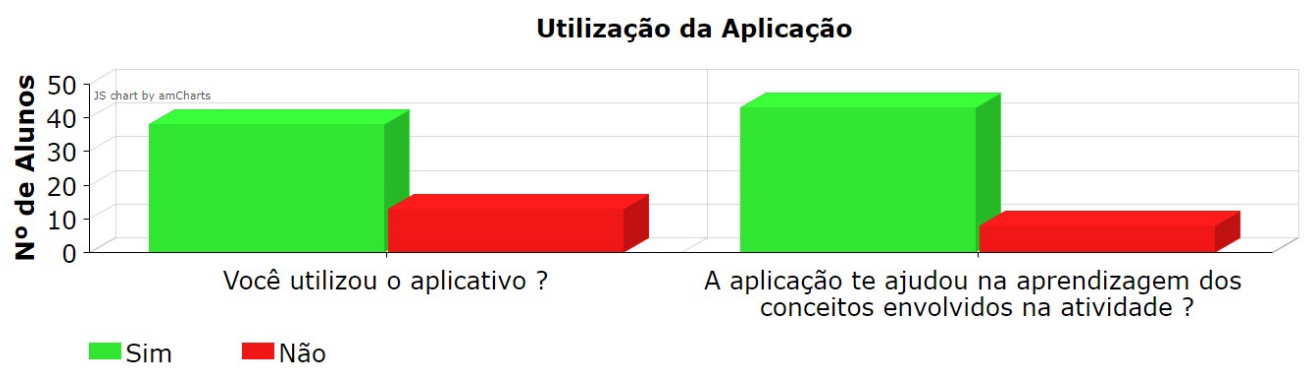

Figure 4. Resultados obtidos com a aplicação do questionário avaliativo, com os 51 alunos do $1^{\circ}$ ano do Ensino Médio.

\section{Considerações Finais}

O desenvolvimento e aplicação da solução proposta neste trabalho permitiu concluir que investir em aplicações tecnológicas é um boa proposta, sendo capaz de contribuir para o processo de ensino-aprendizagem de Física. Além disso, essas soluções tornam o processo mais atrativo e dinâmico para os alunos.

A utilização do aplicativo durante as atividades propostas permitiu também aos professores envolvidos analisarem as principais dificuldades apresentadas pelos alunos e proporem estratégias para corrigi-las quando necessário. Como trabalho futuro pretendese avaliar o aplicativo em um número maior de alunos e permitir aos professores a inserção de material teórico para os alunos por meio do aplicativo.

\section{References}

[Azuma 1997] Azuma, R. T. (1997). A survey of augmented reality. Presence: Teleoperators \& Virtual Environments, 6(4):355-385.

[Barroso and Falcão 2004] Barroso, M. F. and Falcão, E. B. (2004). Evasão universitária: o caso do instituto de física da ufrj. IX Encontro Nacional de Pesquisa em Ensino de Física, 9:1-14.

[Blender 2018] Blender (2018). Blender about @ONLINE.

[Dantas et al. 2017] Dantas, A. C., de Melo, S., Prado, N., Fernandes, M., Takahashi, E., and do Nascimento, M. Z. (2017). Xquestion: Um aplicativo de perguntas e respostas para decisões estratégicas do professor durante uma aula. In Anais dos Workshops do Congresso Brasileiro de Informática na Educação, volume 6, page 160.

[Kaufmann and Meyer 2008] Kaufmann, H. and Meyer, B. (2008). Simulating educational physical experiments in augmented reality. ACM.

[McDermott 2001] McDermott, L. C. (2001). Oersted medal lecture 2001:"physics education research-the key to student learning". American Journal of Physics, 69(11):1127-1137.

[Milgram and Kishino 1994] Milgram, P. and Kishino, F. (1994). A taxonomy of mixed reality visual displays. IEICE TRANSACTIONS on Information and Systems, 77(12):1321-1329.

[SketchUp 2018] SketchUp (2018). Sketchup @ONLINE. 
VIII Congresso Brasileiro de Informática na Educação (CBIE 2019)

Anais dos Workshops do VIII Congresso Brasileiro de Informática na Educação (WCBIE 2019)

[Unity 2018] Unity (2018). Unity documentation @ ONLINE.

[Vuforia 2018] Vuforia (2018). Vuforia developer library - getting started - ar features @ONLINE.

[Wieman and Perkins 2005] Wieman, C. and Perkins, K. (2005). Transforming physics education. Physics today, 58(11):36. 